\title{
Some inequalities related to Sobolev norms
}

\author{
Hoai-Minh Nguyen
}

Received: 10 May 2010 / Accepted: 15 September 2010 / Published online: 12 October 2010

C Springer-Verlag 2010

\begin{abstract}
In this paper, we study some properties related to the new characterizations of Sobolev spaces introduced in Bourgain and Nguyen (C R Acad Sci, 343:75-80, 2006), Nguyen (J Funct Anal 237: 689-720, 2006; J Eur Math Soc 10:191-229, 2008). More precisely, we establish variants of the Poincare inequality, the Sobolev inequality, and the Rellich-Kondrachov compactness theorem, where $\int_{\mathbb{R}^{N}}|\nabla g|^{p} d x$ is replaced by some quantity of the type
\end{abstract}

$$
I_{\delta}(g)=\int_{\substack{\mathbb{R}^{N} \\|g(x)-g(y)|>\delta}} \int_{\mathbb{R}^{N}} \frac{\delta^{p}}{|x-y|^{N+p}} d x d y .
$$

Mathematics Subject Classification (2000) $\quad$ 26D10 - 26A54 - 26D20 - 26A24 .

$26 \mathrm{~A} 33 \cdot 42 \mathrm{~B} 25$

\section{Introduction}

We first introduce the quantity $I_{\delta}(g)$, which plays an important role in this paper,

$$
I_{\delta}(g)=\int_{\substack{\mathbb{R}^{N} \\|g(x)-g(y)|>\delta}} \frac{\delta^{p}}{|x-y|^{N+p}} d x d y \quad \forall g \in L_{\mathrm{loc}}^{1}\left(\mathbb{R}^{N}\right) .
$$

We next recall some new characterizations of Sobolev spaces in $[2,16,18]$. The first one is as follows

Communicated by H. Brezis.

H.-M. Nguyen $(\varangle)$

Courant Institute, NYU, 251 Mercer Street, New York, NY 10012, USA

e-mail: hoaiminh@cims.nyu.edu 
Proposition 1 Let $1<p<+\infty$. Then

(a) There exists a constant $C_{N, p}$ depending only on $N$ and $p$ such that

$$
I_{\delta}(g) \leq C_{N, p} \int_{\mathbb{R}^{N}}|\nabla g|^{p} d x, \quad \forall \delta>0, \quad \forall g \in W^{1, p}\left(\mathbb{R}^{N}\right) .
$$

(b) If $g \in L^{p}\left(\mathbb{R}^{N}\right)$ satisfies

$$
\liminf _{\delta \rightarrow 0_{+}} I_{\delta}(g)<+\infty
$$

then $g \in W^{1, p}\left(\mathbb{R}^{N}\right)$.

(c) Moreover, for any $g \in W^{1, p}\left(\mathbb{R}^{N}\right)$,

$$
\lim _{\delta \rightarrow 0_{+}} I_{\delta}(g)=\frac{1}{p} K_{N, p} \int_{\mathbb{R}^{N}}|\nabla g|^{p} d x,
$$

where $K_{N, p}$ is defined by

$$
K_{N, p}=\int_{\mathbb{S}^{N-1}}|e \cdot \sigma|^{p} d \sigma
$$

for any $e \in \mathbb{S}^{N-1}$.

Remark 1 Assertions (a) and (c) are proved in [16] (Theorem 2). Assertion (b) is proved by Bourgain and Nguyen in [2]. The proof of Assertion (b) is delicate. Under the following stronger assumption

$$
\limsup _{\delta \rightarrow 0_{+}} I_{\delta}(g)<+\infty,
$$

a simple proof is given in [16] (see the proof of Theorem 2).

In [18], we improve statement (b) in Proposition 1 by proving

Proposition 2 Let $N \geq 1, p>1$, and $g \in L^{p}\left(\mathbb{R}^{N}\right)$. Assume that $I_{\delta}(g)<+\infty$ for all $\delta>0$, and

$$
\liminf _{\delta \rightarrow 0_{+}} \int_{\substack{\mathbb{R}^{N} \\ \delta<|g(x)-g(y)|<10 \delta}} \int_{\mathbb{R}^{N}} \frac{\delta^{p}}{|x-y|^{N+p}} d x d y<+\infty .
$$

Then $g \in W^{1, p}\left(\mathbb{R}^{N}\right)$.

Remark 2 To prove Proposition 2, we developed the method introduced in [2]. The observation used in the proof Proposition 2 will play an important role in the proof of statement (b) in Theorem 1 which is crucial to establish Theorem 3 and Proposition 6.

The second characterization is a generalization of Proposition 1.

Proposition 3 [18, Theorem 1] Let $1<p<+\infty$ and $\left(F_{n}\right)_{n \in \mathbb{N}}$ be a sequence of functions from $[0,+\infty)$ into $[0,+\infty)$ such that

(i) $F_{n}(t)$ is a non-decreasing function with respect to $t$ on $[0,+\infty)$, for all $n \in \mathbb{N}$. 
(ii) $\int_{0}^{1} F_{n}(t) t^{-(p+1)} d t=1$, for all $n \in \mathbb{N}$.

(iii) $F_{n}(t)$ converges uniformly to 0 on every compact subset of $(0,+\infty)$ as $n$ goes to infinity.

Then

(a) If $g \in W^{1, p}\left(\mathbb{R}^{N}\right)$, then for every $n \in \mathbb{N}$,

$$
\int_{\mathbb{R}^{N}} \int_{\mathbb{R}^{N}} \frac{F_{n}(|g(x)-g(y)|)}{|x-y|^{N+p}} d x d y \leq C_{N, p} \int_{0}^{\infty} F_{n}(t) t^{-(p+1)} d t \int_{\mathbb{R}^{N}}|\nabla g|^{p} d x,
$$

where $C_{N, p}$ is a positive constant depending only on $N$ and $p$.

(b) If $g \in L^{p}\left(\mathbb{R}^{N}\right)$ and $g$ satisfies

$$
\liminf _{n \rightarrow \infty} \int_{\mathbb{R}^{N}} \int_{\mathbb{R}^{N}} \frac{F_{n}(|g(x)-g(y)|)}{|x-y|^{N+p}} d x d y<+\infty,
$$

then $g \in W^{1, p}\left(\mathbb{R}^{N}\right)$ and

$$
\liminf _{n \rightarrow \infty} \int_{\mathbb{R}^{N}} \int_{\mathbb{R}^{N}} \frac{F_{n}(|g(x)-g(y)|)}{|x-y|^{N+p}} d x d y \geq K_{N, p} \int_{\mathbb{R}^{N}}|\nabla g|^{p} d x .
$$

(c) Moreover, if

$$
\limsup _{n \rightarrow \infty} \int_{0}^{\infty} F_{n}(t) t^{-(p+1)} d t<+\infty
$$

then, for any $g \in W^{1, p}\left(\mathbb{R}^{N}\right)$,

$$
\lim _{n \rightarrow \infty} \int_{\mathbb{R}^{N}} \int_{\mathbb{R}^{N}} \frac{F_{n}(|g(x)-g(y)|)}{|x-y|^{N+p}} d x d y=K_{N, p} \int_{\mathbb{R}^{N}}|\nabla g|^{p} d x .
$$

Here $K_{N, p}$ is defined by (1.1).

Remark 3 Proposition 1 follows from Proposition 3 by choosing

$$
F_{n}(t)= \begin{cases}0 & \text { if } 0 \leq t \leq \delta_{n}, \\ \frac{p \delta_{n}^{p}}{1-\delta_{n}^{p}} & \text { otherwise. }\end{cases}
$$

Remark 4 Assumption (i)-(iii) of the sequence $\left(F_{n}\right)$ are necessary to obtain (a)-(c) (see [18, Remark 4] for detailed discussion).

In this paper, we establish variants of the Poincaré inequality, the Sobolev inequality, and the Rellich-Kondrachov theorem which are inspired by these characterizations. Our first result motivated by Proposition 1 and the Poincaré inequality is the following theorem, which is proved in Sect. 2. 
Theorem 1 Let $N \geq 1, p \geq 1$, and $g$ be a real measurable function defined on a ball $B \subset \mathbb{R}^{N}$. Assume that

$$
\int_{\substack{B \\ g(x)-g(y) \mid>\delta}} \frac{\delta^{p}}{|x-y|^{N+p}} d x d y<+\infty .
$$

Then

(a) If $p \geq 1$, we have

$$
\begin{aligned}
& \int_{B} \int_{B}|g(x)-g(y)|^{p} d x d y \leq C_{N, p} \\
& \quad \times\left(|B|^{\frac{N+p}{N}} \int_{\substack{B \\
|g(x)-g(y)|>\delta}} \frac{\delta}{|x-y|^{N+p}} d x d y+\delta^{p}|B|^{2}\right),
\end{aligned}
$$

for all $\delta>0$ and for some positive constant $C_{N, p}$ depending only on $N$ and $p$.

(b) If $p>1$, we have

$$
\begin{aligned}
& \int_{B} \int_{B}|g(x)-g(y)|^{p} d x d y \leq C_{N, p} \\
& \times\left(|B|^{\frac{N+p}{N}} \int_{\substack{B \\
\delta<|g(x)-g(y)|<10 \delta}} \frac{\delta^{p}}{|x-y|^{N+p}} d x d y+\delta^{p}|B|^{2}\right),
\end{aligned}
$$

for all $\delta>0$. Here $C_{N, p}$ is a positive constant depending only on $N$ and $p$.

Remark 5 We do not know whether (1.4) is valid with $p=1$.

Remark 6 Inequality (1.4) plays an important role in the proof of Theorem 3 below.

Remark 7 A variant of estimate (1.3) was established by Bourgain et al. [4] as follows. Let $g \in C(I=[0,1], \mathbb{R})$. Then

$$
\int_{I} \int_{I}|g(x)-g(y)| d x d y \leq C\left(|I|^{2} \int_{\substack{I \\\left|e^{i g(x)}-e^{i g(y)}\right|>\delta}} \frac{1}{|x-y|^{2}} d x d y+|I|^{2}\right),
$$

for some universal positive constant $C$, when $\delta$ is small. The continuity of $g$ is necessary for such a result. Recently in a joint work with Brezis [6], using a completely different argument, we establish the above inequality for any $\delta<\sqrt{3}$ and for any $g \in \operatorname{VMO}(I, \mathbb{R})(\sqrt{3}$ is optimal). The proofs in [4] and [6] are involved. The approach in [6] can also be used to obtain a similar inequality for $p>1$. These results can be extended to higher dimensions for 
a smooth function $g$ using the idea in Step 2 of the proof of Theorem 1 in this paper (see [7]). Nevertheless we do not know how to obtain (1.3) under the general condition as in statement (a) using this approach since the standard density arguments do not work in this context. This is due to the fact that quantity in the RHS of (1.3) is "unstable" under the convolution.

Our next result is a variant of Rellich-Kondrachov theorem, whose proof is presented in Sect. 3.

Theorem 2 Let $N \geq 1, p \geq 1,\left(g_{n}\right): \mathbb{R}^{N} \rightarrow \mathbb{R}$ be a bounded sequence of functions in $L^{p}\left(\mathbb{R}^{N}\right)$ and $\left(\delta_{n}\right)$ be a sequence of positive numbers converging to 0 such that

$$
\liminf _{n \rightarrow \infty} I_{\delta_{n}}\left(g_{n}\right)=\liminf _{n \rightarrow \infty} \int_{\substack{\mathbb{R}^{N} \\\left|g_{n}(x)-g_{n}(y)\right|>\delta_{n}}} \frac{\delta_{n}^{p}}{|x-y|^{N+p}} d x d y<+\infty .
$$

Then there exist a subsequence $\left(g_{n_{k}}\right)$ of $\left(g_{n}\right)$ and $g \in L^{p}\left(\mathbb{R}^{N}\right)$ such that $\left(g_{n_{k}}\right)$ converges to $g$ in $L_{\mathrm{loc}}^{p}\left(\mathbb{R}^{N}\right)$. Moreover, $g \in W^{1, p}\left(\mathbb{R}^{N}\right)$ for $p>1$ resp. $g \in B V\left(\mathbb{R}^{N}\right)$ for $p=1$ and there exists a positive constant $C$, depending only on $N$ and $p$, such that

$$
\int_{\mathbb{R}^{N}}|\nabla g|^{p} d x \leq C \liminf _{n \rightarrow \infty} I_{\delta_{n}}\left(g_{n}\right) .
$$

Remark 8 The optimal constant in (1.6), which was discussed in the context of Gamma-convergence in $[17,19]$, is strictly less than $K_{N, p} / p$.

Remark 9 The conclusion of Theorem 2 still holds in the case $p>1$ if (1.5) is replaced by the conditions that $I_{\delta_{n}}\left(g_{n}\right)<+\infty$ for all $n \in \mathbb{N}$, and

$$
\liminf _{n \rightarrow \infty} \int_{\substack{\mathbb{R}^{N} \\ \delta_{n}<\left|g_{n}(x)-g_{n}(y)\right|<10 \delta_{n}}} \frac{\delta_{n}^{p}}{|x-y|^{N+p}} d x d y<+\infty .
$$

Remark 10 When $p>1$, Theorem 2 implies the well-known Rellich-Kondrachov theorem, since $I_{\delta}(g) \leq C_{N, p} \int_{\mathbb{R}^{N}}|\nabla g|^{p} d x$.

A variant of the Sobolev inequality, which is proved in Sect. 4, is as follows

Theorem 3 Let $1<p<N, \delta>0$, and $g$ be a real measurable function defined on $\mathbb{R}^{N}$ such that

$$
I_{\delta}(g)=\int_{\substack{\mathbb{R}^{N} \\|g(x)-g(y)|>\delta}} \frac{\delta^{p}}{|x-y|^{N+p}} d x d y<+\infty .
$$

Then there exist two positive constants $C$ and $\lambda$, depending only on $N$ and $p$, such that

$$
\left(\int_{|g|>\lambda \delta}|g|^{q} d x\right)^{\frac{1}{q}} \leq C\left[I_{\delta}(g)\right]^{\frac{1}{p}},
$$

with $q=\frac{N p}{N-p}$. 
Remark 11 Letting $\delta$ go to 0 in (1.7), we rediscover and extend the Sobolev inequality since $\lim _{\delta \rightarrow 0} I_{\delta}(g)=\frac{1}{p} K_{N, p} \int_{\mathbb{R}^{N}}|\nabla g|^{p} d x, I_{\delta}(g) \leq C_{N, p} \int_{\mathbb{R}^{N}}|\nabla g|^{p} d x$ (see Proposition 1), and $\lim _{\delta \rightarrow 0} \int_{|g|>\lambda \delta}|g|^{q} d x=\int_{\mathbb{R}^{N}}|g|^{q} d x$ for $g \in W^{1, p}\left(\mathbb{R}^{N}\right)$. Since $I_{\delta}(g) \leq \frac{\delta^{p}}{\delta^{\prime p}} I_{\delta^{\prime}}(g)$ for $\delta \geq \delta^{\prime}$, Theorem 3 is more interesting when it is applied for large $\delta$.

When $N \geq 1$ and $p=N$, estimates (1.3) and (1.4) clearly imply that $g \in B M O\left(\mathbb{R}^{N}\right)$, the space of all functions of bounded mean oscillation defined on $\mathbb{R}^{N}$ if $g \in L^{1}\left(\mathbb{R}^{N}\right)$ and $I_{\delta}(g)<+\infty$ for some $\delta>0$. Moreover, there exists a positive constant $C$, depending only on $N$, such that

$$
|g|_{\text {BMO }}:=\sup _{Q} \int_{Q} f_{Q}|g(x)-g(y)| d x d y \leq C\left(I_{\delta}^{\frac{1}{N}}(g)+\delta\right),
$$

where the supremum is taken over all cubes of $\mathbb{R}^{N}$. In a joint work with Brezis [7] we also show that if $g \in L^{1}\left(\mathbb{R}^{N}\right)$ and $I_{\delta}(g)<+\infty(p=N)$ for all $\delta>0$, then $g \in \operatorname{VMO}\left(\mathbb{R}^{N}\right)$, the spaces of all functions of vanishing mean oscillation. More properties in the case $p=N$ can be found in [7]. When $p>N$ and $I_{\delta}(g)<+\infty$ for some $\delta$, one cannot hope that $g \in L_{\text {loc }}^{\infty}\left(\mathbb{R}^{N}\right)$. This follows from the fact that the function $g(x):=\ln \ln |\ln | x||$ in $B_{\lambda}(\lambda$ is small), the ball centered at the origin with radius $\lambda$, does not belong to $L^{\infty}\left(B_{\lambda}\right)$ and

$$
\iint_{\substack{B_{\lambda} \\ B_{\lambda} \\ g(x)-g(y) \mid>\delta}} \frac{\delta^{r}}{|x-y|^{N+r}} d x d y<+\infty \quad \forall r>1 .
$$

Applying Theorem 1, we can prove that the sharp function of $g$ belongs to $L_{w}^{q}\left(\mathbb{R}^{N}\right)$ with $q=N p /(N-p)$ if $g \in L^{p}\left(\mathbb{R}^{N}\right)(p \geq 1)$ and $I_{\delta}(g)<+\infty$ for some $\delta>0$ (see Sect. 4). In fact we can prove that $g \in L^{q}\left(\mathbb{R}^{N}\right)$ if $p>1$ and $I_{\delta}(g)<+\infty$ for some $\delta>0$ (see Theorem 3). However, we have the following

Open question 1 Let $p=1$ and $N \geq 2$. Is it true that $g \in L^{\frac{N}{N-1}}\left(\mathbb{R}^{N}\right)$ if $g \in L^{1}\left(\mathbb{R}^{N}\right)$ and $I_{\delta}(g)<+\infty$ for some $\delta>0$ ?

Motivated by Proposition 3, we establish the following results, whose proofs are presented in Sect. 5.

Proposition 4 Let $g$ be a real measurable function defined on a ball $B \subset \mathbb{R}^{N}$ and $F$ : $[0,+\infty) \rightarrow[0,+\infty)$ be a non-decreasing function. Then there exists a constant $C>0$, depending only on $N$ and $p$, such that

$$
\begin{aligned}
& \left(F(1)+\int_{0}^{1} F(t) t^{-(p+1)} d t\right) \int_{B} \int_{B}|g(x)-g(y)|^{p} d x d y \\
& \leq C\left(|B|^{\frac{N+p}{N}} \int_{B} \int_{B} \frac{F(|g(x)-g(y)|)}{|x-y|^{N+p}} d x d y+F(1)|B|^{2}\right) .
\end{aligned}
$$

Proposition 5 Let $1 \leq p<N,\left(F_{n}\right):[0,+\infty) \rightarrow[0,+\infty)$ be a sequence of nondecreasing functions such that $\lim _{n \rightarrow \infty} F_{n}(1)=0$,

$$
F_{n}(1)+\int_{0}^{1} F_{n}(t) t^{-(p+1)} d t=1
$$


and $\left(g_{n}\right): \mathbb{R}^{N} \rightarrow \mathbb{R}$ be a bounded sequence of real functions in $L^{p}\left(\mathbb{R}^{N}\right)$. Assume that

$$
\liminf _{n \rightarrow \infty} \int_{\mathbb{R}^{N}} \int_{\mathbb{R}^{N}} \frac{F_{n}\left(\left|g_{n}(x)-g_{n}(y)\right|\right)}{|x-y|^{N+p}} d x d y<+\infty .
$$

Then there exist a subsequence $\left(g_{n_{k}}\right)$ of $\left(g_{n}\right)$ and $g \in L^{p}\left(\mathbb{R}^{N}\right)$ such that $\left(g_{n_{k}}\right)$ converges to $g$ in $L_{\mathrm{loc}}^{p}\left(\mathbb{R}^{N}\right)$.

Proposition 6 Let $1<p<N, F:[0,+\infty) \rightarrow[0,+\infty)$ be a non-decreasing function and $g$ be a real measurable function defined on $\mathbb{R}^{N}$. Assume that

$$
\int_{\mathbb{R}^{N}} \int_{\mathbb{R}^{N}} \frac{F(|g(x)-g(y)|)}{|x-y|^{N+p}} d x d y<+\infty .
$$

Then there exist two positive constants $C$ and $\lambda$, depending only on $N$ and $p$, such that

$$
\left(\int_{|g|>\lambda F\left(2^{-n}\right)}|g|^{q} d x\right)^{\frac{1}{q}} \leq C\left(\frac{1}{2^{n p} F\left(2^{-n}\right)} \int_{\mathbb{R}^{N}} \int_{\mathbb{R}^{N}} \frac{F(|g(x)-g(y)|)}{|x-y|^{N+p}} d x d y\right)^{\frac{1}{p}} \forall n \in \mathbb{Z},
$$

with $q=\frac{N p}{N-p}$.

Applications of Propositions 4, 5, and 6 will be given in Sect. 5.3. It would be nice to obtain similar results to Theorems 1,2, and 3, and Propositions 4, 5, and 6 in a more general setting e.g. in Carnot-Carathéodory spaces or in metric spaces with appropriate properties.

Recently many authors have suggested various definitions of Sobolev spaces and studied the well-known properties of Sobolev spaces in their contexts e.g. Ambrosio [1], Korevaar and Schoen [14], Reshetnyak [21], Hajlaz and Koskela [12], Bourgain et al. [3] and references therein. The characterizations mentioned in this paper are quite close to the work of Bourgain et al. [3]. However the connection is not transparent.

Theorem 1, whose proof is presented in Sect. 2, is the starting point of this paper. In the proof of Theorem 1, we use of ideas in [2] and [18], and the John and Nirenberg inequality [13]. Theorem 2 is derived from Theorem 1 by the standard technique used in Bourgain etal. [3] (see also [20]). The main ingredient of the proof of Theorem 3 is part (b) of Theorem 1. The proof also makes use of the theory of sharp functions due to Fefferman and Stein [9] and the method of truncation due to Mazya [15]. Obtaining Sobolev's inequality from Poincaré's inequality previously appeared in the literature see e.g. [10-12,22]. However, our approach is different from the works mentioned here, which were inspired by the Riesz potential theory. Moreover, we could not apply their methods in our setting because of the presence of the two terms in the RHS of (1.3) and (1.4). Proposition 4 is derived from Theorem 1 using ideas in [18]. The proofs of Propositions 5 and 6 follow from Proposition 4 by applying the same methods used in the proofs of Theorems 2 and 3.

The paper is organized as follows. In Sect. 2, we prove Theorem 1. Section 3 is devoted to the proof of Theorem 2. Theorem 3 is proved in Sect. 4. Section 5 is devoted to the proofs of Propositions 4, 5, and 6, and their applications. 


\section{A variant of Poincaré's inequality: Proof of Theorem 1}

\subsection{Preliminaries}

In this section, we present some technical lemmas which will be used in the proof of Theorem 1. We first recall some useful results in [18].

Lemma 1 [18, Lemma 3] Let $g$ be a real measurable function defined on the interval $[a, b]$ $(-\infty<a<b<+\infty), z \in \mathbb{R}$, and $\delta>0$. Set

$$
B=\{x \in[a, b] ; g(x)<z\} .
$$

Assume that

$$
0<\frac{|[a, b] \cap B|}{b-a}<1
$$

and

$$
\int_{\substack{a \\ g(x)-g(y) \mid>\delta}}^{b} \frac{1}{|x-y|^{2}} d x d y<+\infty .
$$

Then

$$
\left|[a, b] \cap A_{\tau}\right|>0, \quad \forall \tau>\delta,
$$

where $A_{\tau}:=\{x \in[a, b] ; z \leq g(x)<z+\tau\}$.

Hereafter $|A|$ denotes the Lebesgue measure of $A$ for any measurable set $A \subset \mathbb{R}^{N}$.

Lemma 2 [18, Lemma 4] Let $g$ be a real measurable function defined on the interval $[a, b]$ $(-\infty<a<b<+\infty), z \in \mathbb{R}, r>0, s>0$, and $\tau>\delta>0$. Set

$$
B=\{x \in \mathbb{R} ; g(x)<z\}, \quad A=\{x \in \mathbb{R} ; z \leq g(x)<z+\tau\} .
$$

Assume that

$$
\frac{|[a, b] \cap B|}{b-a}=r, \quad \frac{|[a, b] \cap A|}{b-a} \leq s, \quad r+s<1,
$$

and

$$
\int_{a}^{b} \int_{a}^{b} \frac{1}{|x-y|^{2}} d x d y<+\infty .
$$

Then there exists a subinterval $[c, d] \subset[a, b](a \leq c<d \leq b)$, such that

$$
\frac{|[c, d] \cap B|}{d-c}=r \text { and } s / 4 \leq \frac{|[c, d] \cap A|}{d-c} \leq s .
$$

Lemma 3 [18, Lemma 5] Let $g$ be a real measurable function defined on the interval $[a, b]$ $(-\infty<a<b<+\infty), z \in \mathbb{R}, \tau>\delta>0$, and $0<\lambda \leq 1 / 2$. Set

$$
\left\{\begin{array}{l}
B_{j}=\{x \in \mathbb{R} ; g(x)<z+j \tau\}, \\
A_{j}=\{x \in \mathbb{R} ; z+j \tau \leq g(x)<z+(j+1) \tau\}, \quad \forall j \in \mathbb{Z} .
\end{array}\right.
$$


Assume that

$$
\frac{\left|[a, b] \cap B_{0}\right|}{b-a}=\lambda, \quad \frac{\left|[a, b] \cap A_{0}\right|}{b-a} \leq \lambda / 4,
$$

and

$$
\int_{\substack{a \\ g(x)-g(y) \mid>\delta}}^{b} \frac{1}{|x-y|^{2}} d x d y<+\infty .
$$

Then for each $r>4 / \lambda$, there exist $m \in \mathbb{Z}_{+}, l_{m} \in \mathbb{Z}$, and $[c, d] \subset[a, b](c<d)$ such that

$$
\left\{\begin{array}{l}
\left|l_{m}\right| \leq 2 m, \\
\frac{\left|[c, d] \cap A_{l_{m}}\right|}{d-c} \frac{\left|[c, d] \cap A_{l_{m}+2}\right|}{d-c} \geq \frac{1}{4}[\lambda /(4 r)]^{m+1}, \\
(d-c) \leq 4^{m}[4 /(\lambda r)]^{\frac{m(m-1)}{2}}(b-a) .
\end{array}\right.
$$

Lemma 4 [18, Corollary 6] Let $1<p<+\infty$ and $0<\lambda_{0} \leq \lambda \leq 1 / 2$. Under the assumptions of Lemma 3 , there exist $m \in \mathbb{Z}_{+}$and $l_{m} \in \mathbb{Z}$ such that

$$
\left|l_{m}\right| \leq 2 m
$$

and

$$
\iint_{\substack{x \in[a, b] \cap A_{l_{m}} \\ y \in[a, b] \cap A_{l_{m}+2}}} \frac{1}{|x-y|^{p+1}} d x d y \geq C_{p, \lambda_{0}} m(b-a)^{1-p},
$$

for some positive constant $C_{p, \lambda_{0}}$ depending only on $p$ and $\lambda_{0}$.

Remark 12 Lemmas 3 and 4, which will be used in the proof of part (b) of Lemma 5, are presented in [18] (see [18, Lemma 5] and [18, Corollary 6]) only for the case $\lambda=1 / 2$. However their proofs are almost the same as the ones of [18, Lemma 5] and [18, Corollary 6]. The details are left to the reader.

The following lemma is one of the main ingredients in the proof of Theorem 1 .

Lemma 5 Let $p \geq 1,0<\tau_{0}<\frac{1}{2}$, and $g$ be a real measurable function defined on a bounded interval I. Suppose that there exist $0<\tau_{0}<\tau<\frac{1}{2}, c_{1}<c_{2}$, and two non-empty sub-intervals $I_{1}$ and $I_{2}$ of I such that

$$
\left|\left\{x \in I_{1} ; g(x)<c_{1}\right\}\right| \geq \tau\left|I_{1}\right| \text { and }\left|\left\{x \in I_{2} ; g(x)>c_{2}\right\}\right| \geq \tau\left|I_{2}\right| .
$$

Then there exists some positive constant $C$ depending only on $p$ and $\tau_{0}$ such that:

(a) If $p \geq 1$, we have

$$
\iint_{\substack{I \\|g(x)-g(y)|>\delta}} \frac{\delta^{p}}{|x-y|^{p+1}} d x d y \geq C_{p, \tau_{0}}\left(c_{2}-c_{1}\right)^{p}|I|^{1-p}, \quad \forall \delta \in\left(0, \delta_{0}\right) .
$$


(b) If $p>1, \delta \in\left(0, \delta_{0}\right)$, and

$$
\iint_{\substack{I \\ I}} \frac{\delta^{p}}{|x-y|^{p+1}} d x d y<+\infty
$$

we have

$$
\int_{\substack{I \\ I}} \int_{\substack{I \\(x)-g(y) \mid<10 \delta}} \frac{\delta^{p}}{|x-y|^{p+1}} d x d y \geq C_{p, \tau_{0}}\left(c_{2}-c_{1}\right)^{p}|I|^{1-p} .
$$

Here $\delta_{0}=\frac{\tau\left(c_{2}-c_{1}\right)}{200} \min \left\{\frac{\left|I_{1}\right|}{|I|}, \frac{\left|I_{2}\right|}{|I|}\right\}$.

Remark 13 Lemma 5 is a variant of [2, Lemma 2] and [18, Lemma 6] stating that the limit of the LHS of (2.2) and (2.3) as $\delta$ goes to 0 gives upper bounds of $|I|^{1-p}\left(\operatorname{ess~sup~}_{I} g-\operatorname{ess~inf}_{I} g\right.$ ) up to a constant. Lemma 5 gives the range of $\delta$ (independent of $g$ ) for which (2.2) and (2.3) hold if (2.1) is satisfied. The proof of Lemma 5 completely borrows arguments used in the ones of [2, Lemma 2] and [18, Lemma 6].

In what follows, the notation $a \lesssim b$ means that there exists a positive constant $c$ depending only on $N$ and $p$, such that $a \leq c b$. The notation $a \gtrsim b$ means that $b \lesssim a$ and the notation $a \approx b$ means that $a \lesssim b$ and $b \lesssim a$.

Proof By scaling and translating, one can assume as well that $I=[0,1], c_{1}=0$, and $c_{2}=1$. Take $\delta \in\left(0, \frac{\tau}{200}\right) \min \left\{\left|I_{1}\right|,\left|I_{2}\right|\right\}$ and $K \in \mathbb{Z}_{+}$such that

$$
\delta<2^{-K} \leq 2 \delta .
$$

Denote

$$
J=\left\{j \in \mathbb{Z}_{+} ; \frac{1}{4}<j 2^{-K}<\frac{3}{4}\right\} .
$$

Then

$$
\operatorname{card}(J) \geq 2^{K-1}-2 \approx \frac{1}{\delta}
$$

For each $j$, define the following sets

$$
\begin{aligned}
& A_{j}=\left\{x \in[0,1] ;(j-1) 2^{-K} \leq g(x)<j 2^{-K}\right\}, \\
& B_{j}=\bigcup_{j^{\prime}<j} A_{j^{\prime}}, \quad \text { and } C_{j}=\bigcup_{j^{\prime}>j} A_{j^{\prime}},
\end{aligned}
$$

so that $B_{j} \times C_{j} \subset\left[|g(x)-g(y)| \geq 2^{-K}\right] \subset[|g(x)-g(y)|>\delta]$.

Set

$$
G=\left\{j \in J ;\left|A_{j}\right|<2^{-K+2}\right\} .
$$

Since the collection $\left(A_{j}\right)$ is disjoint, it follows from (2.5) that

$$
\operatorname{card}(G) \geq 2^{K-2}-3 \approx \frac{1}{\delta}
$$


For each $j \in G$, set $\lambda_{1, j}=\left|A_{j}\right|>0$ by Lemma 1 . We claim that there exist $s_{1, j}$ and $s_{2, j}$ in $\left[4 \lambda_{1, j}, 1-4 \lambda_{1, j}\right]$ such that

$$
\begin{aligned}
& \left|\left[s_{1, j}-4 \lambda_{1, j}, s_{1, j}+4 \lambda_{1, j}\right] \cap B_{j}\right|>\tau / 2 \text { and }\left|\left[s_{2, j}-4 \lambda_{1, j}, s_{2, j}+4 \lambda_{1, j}\right] \cap\left(A_{j} \cup C_{j}\right)\right| \\
& \quad>\tau / 2 .
\end{aligned}
$$

We first prove that there exists $s_{1, j} \in\left[4 \lambda_{1, j}, 1-4 \lambda_{1, j}\right]$ such that

$$
\left|\left[s_{1, j}-4 \lambda_{1, j}, s_{1, j}+4 \lambda_{1, j}\right] \cap B_{j}\right|>\tau / 2
$$

by contradiction. Suppose that

$$
\left|\left[t-4 \lambda_{1, j}, t+4 \lambda_{1, j}\right] \cap B_{j}\right|<\tau / 2 \quad \forall t \in\left[4 \lambda_{1, j}, 1-4 \lambda_{1, j}\right] .
$$

Set $t_{0}=4 \lambda_{1, j}+\inf _{x \in I_{1}} x$ and $t_{i+1}=t_{i}+8 \lambda_{1, j}$ for $i \geq 0$. Let $n$ be such that $t_{n}+4 \lambda_{1, j} \in I_{1}$ and $t_{n+1}+4 \lambda_{1, j} \notin I_{1}$. We have

$$
\left|I_{1} \cap B_{j}\right| \leq \sum_{i=0}^{n}\left|\left[t_{i}-4 \lambda_{1, j}, t_{i}+4 \lambda_{1, j}\right] \cap B_{j}\right|+8 \lambda_{1, j} .
$$

We deduce from (2.10) that

$$
\left|I_{1} \cap B_{j}\right| \leq \tau\left|I_{1}\right| / 2+8 \lambda_{1, j}
$$

However since $j \in G, 2^{-K} \leq \delta \leq \tau\left|I_{1}\right| / 200$, it follows from (2.6) that

$$
8 \lambda_{1, j} \leq 8.2^{-K+2}=32.2^{-K} \leq 64 \delta<\tau\left|I_{1}\right| / 2 .
$$

Combining (2.11) and (2.12) yields that

$$
\left|I_{1} \cap B_{j}\right|<\tau\left|I_{1}\right| .
$$

This contradicts the fact that $\left|I_{1} \cap B_{j}\right| \geq\left|I_{1} \cap B_{1}\right|>\tau\left|I_{1}\right|$.

Thus there exists $s_{1, j} \in\left[4 \lambda_{1, j}, 1-4 \lambda_{1, j}\right]$ such that $\left|\left[s_{1, j}-4 \lambda_{1, j}, s_{1, j}+4 \lambda_{1, j}\right] \cap B_{j}\right|>$ $\tau / 2$.

Similarly, since $\left|I_{2} \cap\left(A_{j} \cup C_{j}\right)\right|>\tau\left|I_{2}\right|$, there exists $s_{2, j} \in\left[4 \lambda_{1, j}, 1-4 \lambda_{1, j}\right]$ such that $\left|\left[s_{2, j}-4 \lambda_{1, j}, s_{2, j}+4 \lambda_{1, j}\right] \cap\left(A_{j} \cup C_{j}\right)\right|>\tau / 2$. Therefore (2.8) is proved.

From (2.8) it is clear that there exists $t_{1, j} \in\left[4 \lambda_{1, j}, 1-4 \lambda_{1, j}\right]$ such that

$$
\tau / 2 \leq\left|\left[t_{1, j}-4 \lambda_{1, j}, t_{1, j}+4 \lambda_{1, j}\right] \cap B_{j}\right| \leq 1-\tau / 2 .
$$

On the other hand, since $\left|A_{j}\right| \leq 2^{-K+2} \leq 8 \delta \leq \tau / 8$, it follows that

$$
\left|\left[t_{1, j}-4 \lambda_{1, j}, t_{1, j}+4 \lambda_{1, j}\right] \cap A_{j}\right| \leq\left|A_{j}\right| \leq \tau / 8 .
$$

Combining (2.13) and (2.14) and using Lemma 2, we have, for some $t_{j}, \lambda_{j}>0$,

$$
\tau / 2 \leq \frac{\left|\left[t_{j}-4 \lambda_{j}, t_{j}+4 \lambda_{j}\right] \cap B_{j}\right|}{8 \lambda_{j}} \leq 1-\tau / 2,
$$

and

$$
\tau / 32 \leq \frac{\left|\left[t_{j}-4 \lambda_{j}, t_{j}+4 \lambda_{j}\right] \cap A_{j}\right|}{8 \lambda_{j}} \leq \tau / 8 .
$$

Proof of $(a): p \geq 1$. Set $\lambda=\inf _{j \in G} \lambda_{j}\left(\lambda>0\right.$ since $G$ is finite). Define $P_{m}$ as follows

$$
P_{m}=\left\{j \in G ; 2^{m-1} \lambda \leq \lambda_{j}<2^{m} \lambda\right\}, \quad \forall m \geq 1 .
$$


Then $G=\bigcup_{i=1}^{n} P_{m}$ for some $n$. From (2.7), we have

$$
\sum_{m=1}^{n} \operatorname{card}\left(P_{m}\right) \gtrsim \frac{1}{\delta}
$$

For each $m(1 \leq m \leq n)$, since $A_{j} \cap A_{k}=\emptyset$ for $j \neq k$, it follows from (2.16) that there exists $J_{m} \subset P_{m}$ such that

$$
\text { (a) } \operatorname{card}\left(J_{m}\right) \gtrsim \operatorname{card}\left(P_{m}\right) \text { and }(b)\left|t_{i}-t_{j}\right|>2^{m+3} \lambda, \quad \forall i, j \in J_{m}, i \neq j .
$$

Combining (2.17), (2.18), and (2.19) yields

$$
\left[t_{i}-4 \lambda_{i}, t_{i}+4 \lambda_{i}\right] \cap\left[t_{j}-4 \lambda_{j}, t_{j}+4 \lambda_{j}\right]=\emptyset, \quad \forall i, j \in J_{m}, i \neq j,
$$

and

$$
\sum_{m=1}^{n} \operatorname{card}\left(J_{m}\right) \gtrsim \frac{1}{\delta}
$$

Set $U_{0}:=\varnothing$ and, for $m=1,2, \ldots, n$,

$$
\left\{\begin{array}{l}
L_{m}=\left\{j \in J_{m} ;\left|\left[t_{j}-4 \lambda_{j}, t_{j}+4 \lambda_{j}\right] \backslash U_{m-1}\right| \geq(8-\tau / 16) \lambda_{j}\right\} \\
U_{m}=\left(\bigcup_{j \in L_{m}}^{\bigcup}\left[t_{j}-4 \lambda_{j}, t_{j}+4 \lambda_{j}\right]\right) \cup U_{m-1}, \\
a_{m}=\operatorname{card}\left(J_{m}\right) \text { and } b_{m}=\operatorname{card}\left(L_{m}\right) .
\end{array}\right.
$$

From (2.20), we have

$$
\sum_{j \in J_{m} \backslash L_{m}}\left|\left[t_{j}-4 \lambda_{j}, t_{j}+4 \lambda_{j}\right]\right| \lesssim \frac{1}{\tau}\left|U_{m-1}\right| .
$$

Hence since $L_{m} \subset J_{m} \subset P_{m}$, it follows from (2.17) that

$$
2^{m-1}\left(a_{m}-b_{m}\right) \lesssim \frac{1}{\tau} \sum_{i=1}^{m-1} 2^{i} b_{i}
$$

which shows that

$$
a_{m} \lesssim b_{m}+\frac{8}{\tau} \sum_{i=1}^{m-1} 2^{(i-m)} b_{i}
$$

Consequently,

$$
\sum_{m=1}^{n} a_{m} \lesssim \sum_{m=1}^{n} b_{m}+\frac{8}{\tau} \sum_{m=1}^{n} \sum_{i=1}^{m-1} 2^{(i-m)} b_{i}=\sum_{m=1}^{n} b_{m}+\frac{8}{\tau} \sum_{i=1}^{n} b_{i} \sum_{m=i+1}^{n} 2^{(i-m)} .
$$

Since $\sum_{i=1}^{\infty} 2^{-i}=1$, it follows from (2.18) and (2.21) that

$$
\sum_{m=1}^{n} b_{m} \gtrsim \tau \sum_{m=1}^{n} a_{m} \gtrsim \frac{\tau}{\delta} .
$$


Combining (2.15), (2.16), (2.22), and (2.23) yields

$$
\begin{aligned}
\iint_{\substack{I \\
|g(x)-g(y)|>\delta}} \frac{\delta^{p}}{|x-y|^{p+1}} d x d y & \geq \sum_{m=1}^{n} \sum_{j \in L_{m}} \iint_{\substack{\left(\left[t_{j}-4 \lambda_{j}, t_{j}+4 \lambda_{j}\right] \backslash U_{m-1}\right)^{2} \\
x \in B_{j}, y \in C_{j}}} \frac{\delta^{p}}{|x-y|^{p+1}} d x d y \\
& \gtrsim \delta^{p} \sum_{m=1}^{n} b_{m} \tau / \delta^{p-1} \gtrsim \tau^{2} .
\end{aligned}
$$

This implies the conclusion of Lemma 5 in this case.

Proof of $(b): p>1$. Take $j \in G$. By Lemma 4, we deduce from (2.15) and (2.16) that there exist $m_{j} \in \mathbb{Z}_{+}$and $l_{j} \in \mathbb{Z}$ such that

$$
\left|l_{j}-j\right| \leq 2 m_{j}
$$

and

$$
\iint_{\substack{x \in \mathcal{I} \cap A_{l_{j}} \\ y \in \mathcal{I} \cap A_{l_{j}+2}}} \frac{1}{|x-y|^{p+1}} d x d y \gtrsim c_{\tau_{0}} m_{j} \lambda_{j}^{1-p} \gtrsim c_{\tau_{0}} m_{j} \delta^{1-p} .
$$

Hereafter $c_{\tau_{0}}$ denotes a positive constant depending only on $\tau_{0}$. The last inequality follows from the fact that $\lambda_{j} \lesssim \delta$.

Set $i_{0}=-1$ and

$$
C_{i}=\left\{j \in G ; l_{j}=i\right\}, \quad \forall i \in \mathbb{Z}
$$

For each $n \geq 1$, if

$$
\left\{i \in \mathbb{Z} ; i \geq i_{n-1}+1 \text { and } C_{i} \neq \varnothing\right\} \neq \varnothing
$$

then set

$$
\left\{\begin{array}{l}
i_{n}=\inf \left\{i \in \mathbb{Z} ; i \geq i_{n-1}+1 \text { and } C_{i} \neq \varnothing\right\}, \\
k_{n}=\max \left\{m_{j} ; j \in G \text { and } l_{j}=i_{n}\right\} .
\end{array}\right.
$$

From (2.24), we have

$$
k_{n} \gtrsim \operatorname{card}\left\{j \in G ; l_{j}=i_{n}\right\} .
$$

Hence we deduce from (2.7) that

$$
\sum_{n \geq 1, k_{n} \text { exists }} k_{n} \gtrsim \operatorname{card}(G) \approx \frac{1}{\delta} .
$$

On the other hand, from (2.25),

$$
\begin{aligned}
\int_{\substack{\mathcal{I} \\
\underbrace{}_{\mathcal{I}}}} \frac{\delta^{p}}{|x-y|^{p-1}} d x d y & \geq \sum_{n \geq 1, k_{n} \text { exists }} \iint_{\substack{x \in \mathcal{I} \cap A_{i_{n}} \\
y \in \mathcal{I} \cap A_{i_{n}+2}}} \frac{\delta^{p}}{|x-y|^{p+1}} d x d y \\
& \gtrsim c_{\tau_{0}} \sum_{n \geq 1, k_{n} \text { exists }} k_{n} \delta
\end{aligned}
$$

Therefore the conclusion of Lemma 4 in the case $p>1$ follows from (2.26), (2.27), and (2.4). 


\subsection{Proof of Theorem 1}

Step 1: $N=1$. Let $I$ be a bounded interval of $\mathbb{R}$. We first assume that $g \in L^{\infty}(I)$ and

$$
\int_{\substack{I \\ I}} \frac{\delta^{p}}{|x-y|^{p+1}} d x d y<+\infty
$$

and prove, if $p \geq 1$,

$$
\int_{I} \int_{I}|g(x)-g(y)|^{p} d x d y \leq C_{p}\left(|I|^{p+1} \int_{\substack{I \\|g(x)-g(y)|>\delta}} \frac{\delta^{p}}{|x-y|^{p+1}} d x d y+\delta^{p}|I|^{2}\right),
$$

and, if $p>1$,

$$
\int_{I} \int_{I}|g(x)-g(y)|^{p} d x d y \leq C_{p}\left(|I|^{p+1} \int_{\substack{I \\ \delta<|g(x)-g(y)|<10 \delta}} \frac{\delta^{p}}{|x-y|^{p+1}} d x d y+\delta^{p}|I|^{2}\right),
$$

where $C_{p}$ is a positive constant depending only on $p$.

By scaling, one may assume that $I=[0,1]$ and

$$
|g|_{B M O(I)}=2 .
$$

We recall the following fact due to John and Nirenberg [13]: There exist two universal constants $c_{1}$ and $c_{2}$ such that if $-\infty<a<b<+\infty$ and $u \in B M O([a, b])$ then

$$
\left|\left\{x \in(a, b) ;\left|u-\int_{a}^{b} u(s) d s\right|>t\right\}\right| \leq c_{1}(b-a) \exp \left(-\frac{c_{2} t}{|u|_{B M O([a, b])}}\right) \quad \forall t>0 .
$$

Let $0<a<b<1$ be such that

$$
f_{a}^{b}\left|g(x)-\int_{a}^{b} g(s) d s\right| d x \geq 1 .
$$

The existence of $a$ and $b$ follows from (2.30). Without loss of generality, one may assume that

$$
\int_{a}^{b} g d x=0
$$

By (2.31), it follows from (2.30), (2.32) and (2.33) that there exist two universal constants $\tau_{1}<0$ and $\tau_{2}>0$ such that

$$
\frac{1}{b-a}\left|\left\{x \in(a, b) ; g(x)<\tau_{1}\right\}\right| \gtrsim 1 \text { and } \frac{1}{b-a}\left|\left\{x \in(a, b) ; g(x)>\tau_{2}\right\}\right| \gtrsim 1 .
$$


Applying Lemma 5, we have, if $p \geq 1$,

$$
\int_{\substack{a \\ g(x)-g(y) \mid>\delta}}^{b} \frac{\delta^{p}}{|x-y|^{p+1}} d x d y+\delta^{p} \gtrsim 1 \quad \forall \delta>0 .
$$

and, if $p>1$,

$$
\int_{\substack{a \\ g(x)-g(y) \mid<10 \delta}}^{b} \frac{\delta^{p}}{|x-y|^{p+1}} d x d y+\delta^{p} \gtrsim 1 \quad \forall \delta>0 .
$$

This completes the proof in the case $g \in L^{\infty}(I)$.

The proof in the general case (without assuming that $g \in L^{\infty}(I)$ ) goes as follows. Let $A>0$ and define

$$
g_{A}=\min \{\max \{g,-A\}, A\} .
$$

Then $g_{A} \in L^{\infty}(I)$. Hence it follows from (2.28) and (2.29) that if $p \geq 1$

$\int_{I} \int_{I}\left|g_{A}(x)-g_{A}(y)\right|^{p} d x d y \leq C_{p}\left(|I|^{p+1} \int_{\substack{I \\|g(x)-g(y)|>\delta}} \frac{\delta^{p}}{|x-y|^{p+1}} d x d y+\delta^{p}|I|^{2}\right) \forall p \geq 1$,

and if $p>1$

$$
\begin{aligned}
& \int_{I} \int_{I}\left|g_{A}(x)-g_{A}(y)\right|^{p} d x d y \leq C_{p}\left(|I|^{p+1} \int_{\substack{I \\
\delta<\left|g_{A}(x)-g_{A}(y)\right|<10 \delta}} \frac{\delta^{p}}{|x-y|^{p+1}} d x d y+\delta^{p}|I|^{2}\right) \\
& \quad \forall p>1 .
\end{aligned}
$$

By letting $A$ go to infinity and using Fatou's lemma, the conclusion follows.

Step 2: $N \geq 2$. Let us sketch the proof in the case $N=2$. The proof in the general case follows similarly. We present here only the proof of (1.4). The proof of (1.3) is almost the same as the one of (1.4). Without loss of generality, one may assume that $B=B_{1}$ the unit ball centered at the origin. Let $f$ be an extension of $g$ on $B_{8}$, the ball centered at 0 with radius 8 , such that

$$
\int_{\substack{B_{1} \\ \delta<|g(x)-g(y)|<10 \delta}} \frac{1}{|x-y|^{N+p}} d x d y \approx \int_{\substack{B_{8} \\ B_{8}}} \int_{\substack{B_{8} \\ \delta<|f(x)-f(y)|<10 \delta}} \frac{1}{|x-y|^{N+p}} d x d y
$$

and

$$
\int_{\substack{B_{1} \\ \mid B_{1}}} \int_{\substack{B_{1} \\ g(x)-g(y) \mid>\delta}} \frac{1}{|x-y|^{N+p}} d x d y \approx \int_{\substack{B_{8} \\|f(x)-f(y)|>\delta}} \int_{B_{8}} \frac{1}{|x-y|^{N+p}} d x d y .
$$


We first note that, with $e_{1}=(1,0)$ and $e_{2}=(0,1)$.

$$
\begin{aligned}
& \left|f\left(s R\left(e_{1}\right)+t R\left(e_{2}\right)\right)-f\left(\hat{s} R\left(e_{1}\right)+\hat{t} R\left(e_{2}\right)\right)\right|^{p} \\
& \quad \lesssim\left|f\left(s R\left(e_{1}\right)+t R\left(e_{2}\right)\right)-f\left(\hat{s} R\left(e_{1}\right)+t R\left(e_{2}\right)\right)\right|^{p}+\mid f\left(\hat{s} R\left(e_{1}\right)+t R\left(e_{2}\right)\right) \\
& \quad-\left.f\left(\hat{s} R\left(e_{1}\right)+\hat{t} R\left(e_{2}\right)\right)\right|^{p},
\end{aligned}
$$

for all $s, t \in \mathbb{R}$ and $R \in S O(2)$ i.e. $R$ is a rotation.

On the other hand, applying Theorem 1 in the case $N=1$, we have

$$
\begin{aligned}
& \int_{-1}^{1} \int_{-1}^{1} \int_{-1}^{1}\left|f\left(s R\left(e_{1}\right)+t R\left(e_{2}\right)\right)-f\left(\hat{s} R\left(e_{1}\right)+t R\left(e_{2}\right)\right)\right|^{p} d s d \hat{s} d t \\
& \lesssim \int_{-1}^{1} \int_{-1}^{1} \int_{-1}^{1} \frac{\delta^{p}}{|s-\hat{s}|^{p+1}} d s d \hat{s} d t+\delta^{p} .
\end{aligned}
$$

This implies

$$
\begin{aligned}
& \int_{-1}^{1} \int_{-1}^{1} \int_{-1}^{1}\left|f\left(s R\left(e_{1}\right)+t R\left(e_{2}\right)\right)-f\left(\hat{s} R\left(e_{1}\right)+t R\left(e_{2}\right)\right)\right|^{p} d s d \hat{s} d t \\
& \lesssim \int_{-2}^{2} \int_{\substack{-2 \\
\delta<\left|f\left(h R\left(e_{1}\right)+x\right)-f(x)\right|<10 \delta}}^{2} \frac{\delta^{p}}{|h|^{p+1}} d x d h+\delta^{p} .
\end{aligned}
$$

Hereafter $x=\left(x_{1}, x_{2}\right)=x_{1} e_{1}+x_{2} e_{2}$. Similarly, we have

$$
\begin{aligned}
& \int_{-1}^{1} \int_{-1}^{1} \int_{-1}^{1}\left|f\left(\hat{s} R\left(e_{1}\right)+t R\left(e_{2}\right)\right)-f\left(\hat{s} R\left(e_{1}\right)+t R\left(e_{2}\right)\right)\right|^{p} d t d \hat{t} d \hat{s} \\
& \lesssim \int_{-2}^{2} \int_{\substack{-2 \\
\delta<\left|f\left(h R\left(e_{2}\right)+x\right)-f(x)\right|<10 \delta}}^{2} \frac{\delta^{p}}{|h|^{p+1}} d x d h+\delta^{p} .
\end{aligned}
$$

Combining (2.35), (2.37), (2.38), and (2.39) yields

$$
\begin{aligned}
& \int_{B_{1}} \int_{B_{1}}|g(x)-g(y)|^{p} d x d y \lesssim \int_{-2}^{2} \int_{\substack{-2 \\
\delta<\left|f\left(h R\left(e_{1}\right)+x\right)-f(x)\right|<10 \delta}}^{2} \frac{\delta^{p}}{|h|^{p+1}} d x d h \\
& +\int_{-2}^{2} \int_{\substack{-2 \\
\delta<\left|f\left(h R\left(e_{2}\right)+x\right)-f(x)\right|<10 \delta}}^{2} \frac{\delta^{p}}{|h|^{p+1}} d x d h+\delta^{p} .
\end{aligned}
$$

Therefore the conclusion follows after integrating two sides of the above inequality with respect to $R$. 


\section{A variant of Rellich-Kondrachov's theorem: Proof of Theorem 2}

In this section, we prove Theorem 2. The following lemma is the key of this section.

Lemma 6 Let $g: \mathbb{R}^{N} \rightarrow \mathbb{R}$ be a real measurable function and $Q$ be a cube of $\mathbb{R}^{N}$. Then

$$
\int_{Q}\left|g(x)-g_{\varepsilon}(x)\right|^{p} d x \lesssim \varepsilon^{p} \int_{\substack{\mathbb{R}^{N} \\|g(x)-g(y)|>\delta}} \frac{\delta^{p}}{|x-y|^{N+p}} d x d y+\delta^{p}|Q|,
$$

where

$$
g_{\varepsilon}=\frac{1}{\left|\varepsilon Q_{1}\right|} g * \chi_{\varepsilon} .
$$

Here $Q_{1}$ is the unit cube centered at the origin and $\chi_{\varepsilon}$ is the characteristic function of $\varepsilon Q_{1}$.

Henceforth $a Q$ denotes the cube with the same center as $Q$ and $a$ times its length for any cube $Q$ of $\mathbb{R}^{N}$.

Proof Let $\left(Q_{i}\right)_{i \in I}$ be a collection of open cubes such that

$$
\left|Q_{i}\right|=\varepsilon^{N}, \quad Q_{i} \cap Q_{j}=\emptyset, \quad \forall i \neq j, \quad \text { and } Q \subset \cup_{i \in I} \overline{Q_{i}} .
$$

Then

$$
\int_{Q}\left|g(x)-g_{\varepsilon}(x)\right|^{p} d x \leq \sum_{i \in I} \int_{Q_{i}}\left|g(x)-g_{\varepsilon}(x)\right|^{p} d x .
$$

Hence since

$$
\int_{Q_{i}}\left|g(x)-g_{\varepsilon}(x)\right|^{p} d x \leq \frac{1}{\varepsilon^{N}} \int_{3 Q_{i}} \int_{3 Q_{i}}|g(x)-g(y)|^{p} d x d y,
$$

it follows that

$$
\int_{Q}\left|g(x)-g_{\varepsilon}(x)\right|^{p} d x \leq \frac{1}{\varepsilon^{N}} \sum_{i} \int_{3 Q_{i}} \int_{3 Q_{i}}|g(x)-g(y)|^{p} d x d y .
$$

Applying Theorem 1, we deduce from (3.1) that

$$
\frac{1}{\varepsilon^{N}} \sum_{i} \int_{3 Q_{i}} \int_{3 Q_{i}}|g(x)-g(y)|^{p} d x d y \leq C_{N, p}\left(\varepsilon^{p} \int_{\substack{\mathbb{R}^{N} \\|g(x)-g(y)|>\delta}} \frac{\delta^{p}}{|x-y|^{N+p}} d x d y+\delta^{p}|Q|\right) \text {. }
$$

Combining (3.2) and (3.3) yields

$$
\int_{Q}\left|g(x)-g_{\varepsilon}(x)\right|^{p} d x \leq C_{N, p}\left(\varepsilon^{p} \int_{\substack{\mathbb{R}^{N} \\|g(x)-g(y)|>\delta}} \frac{\delta^{p}}{|x-y|^{N+p}} d x d y+\delta^{p}|Q|\right) .
$$


We are ready to prove Theorem 2. We follow the standard approach used in [3] (see also [20]).

Proof of Theorem 2 Applying Lemma 6, we have, for each cube $Q$ of $\mathbb{R}^{N}$,

$$
\int_{Q}\left|g_{n}(x)-g_{n, \varepsilon}(x)\right|^{p} d x \leq C_{N, p}\left(\varepsilon^{p} \int_{\substack{\mathbb{R}^{N} \\ \mathbb{R}^{N}\left(g_{n}(x)-g_{n}(y) \mid>\delta_{n}\right.}} \frac{\delta_{n}^{p}}{|x-y|^{N+p}} d x d y+\delta_{n}^{p}|Q|\right),
$$

where

$$
g_{n, \varepsilon}=\frac{1}{|\varepsilon Q|} g_{n} * \chi_{\varepsilon} .
$$

Here $Q_{1}$ is the unit cube centered at the origin and $\chi_{\varepsilon}$ is the characteristic function of $\varepsilon Q_{1}$. Hence

$$
\lim _{\varepsilon \rightarrow 0}\left(\limsup _{n \rightarrow \infty} \int_{Q}\left|g_{n}(x)-g_{n, \varepsilon}(x)\right|^{p} d x\right)=0 .
$$

Thus, since $\left(g_{n}\right)$ is bounded in $L^{p}\left(\mathbb{R}^{N}\right)$, by the theorem of Riesz et al. (see e.g. [5, Theorem IV.25]) and [5, Corollary IV.27], there exists a sub-sequence $\left(g_{n_{k}}\right)$ of $\left(g_{n}\right)$ and $g \in L^{p}\left(\mathbb{R}^{N}\right)$ such that $g_{n_{k}}$ converges to $g$ in $L_{\text {loc }}^{p}\left(\mathbb{R}^{N}\right)$. The second assertion of Theorem 2 follows from [18, Theorem 3].

\section{A variant of Sobolev's inequality: Proof of Theorem 3}

This section will be devoted to the proof of Theorem 3. One of the main ingredients of the proof is the estimate in part (b) of Theorem 1. The proof also makes use of the theory of sharp functions and the truncation method.

We first recall the definition of the dyadic maximal function $M^{\Delta} g$ and the dyadic sharp function $g \sharp, \Delta$ associated with $g$ (see e.g. [24]).

Definition 1 Let $g \in L_{\text {loc }}^{1}\left(\mathbb{R}^{N}\right)$. Then $M^{\Delta} g$ and $g^{\sharp, \Delta}$ are defined as follows

$$
\left(M^{\Delta} g\right)(x)=\sup _{Q} \int_{Q}|g| d y,
$$

and

$$
g^{\sharp, \Delta}(x)=\sup _{Q} \int_{Q}\left|g-g_{Q}\right| d y,
$$

where the supremum is taken over all dyadic cubes $Q$ containing $x$ and $g_{Q}:=f_{Q} g d y$.

The following result which is a consequence of Vitali's covering theorem will be used in the proof of Theorem 3 . 
Lemma 7 Let $\delta>0,0<\theta_{1}<\theta_{2}, h \in L^{1}\left(\mathbb{R}^{N}\right)$, and $g$ be a real measurable function such that

$$
g(x) \leq \sup _{B}|B|^{\theta_{1}}\left(f_{B}|h| d x\right)^{\theta_{2}}+\delta, \quad \forall x \in \mathbb{R}^{N},
$$

where the supremum is taken over all balls $B$ containing $x$. Then

$$
|\{g>t\}| \leq C\|h\|_{L^{1}}^{\frac{\theta_{2}}{\theta_{2}-\theta_{1}}} / t^{\frac{1}{\theta_{2}-\theta_{1}}}, \quad \forall t>2 \delta,
$$

for some positive constant $C$, depending only on $\theta_{1}$ and $\theta_{2}$.

Proof Let $t>2 \delta$. For each $y \in\{g>t\}$, from (4.2), there exists a ball $B_{y}$ containing $y$ such that

$$
t \leq 2\left|B_{y}\right|^{\theta_{1}}\left(f_{B_{y}}|h| d x\right)^{\theta_{2}} .
$$

It follows that

$$
\left|B_{y}\right|^{\theta_{2}-\theta_{1}} \leq \frac{2}{t}\left(\int_{B_{y}}|h| d y\right)^{\theta_{2}}
$$

which implies

$$
\left|B_{y}\right| \leq \frac{C}{t^{\frac{1}{\theta_{2}-\theta_{1}}}}\left(\int_{B_{y}}|h| d y\right)^{\frac{\theta_{2}}{\theta_{2}-\theta_{1}}}<+\infty,
$$

since $h \in L^{1}\left(\mathbb{R}^{N}\right)$. Hereafter in the proof, $C$ denotes a positive constant depending only on $\theta_{1}$ and $\theta_{2}$. Applying Vitali's covering theorem (see e.g. [8]), there exists a denumerable collection of balls $\left(B_{i}\right)$ such that $B_{i} \cap B_{j}=\emptyset$ for $i \neq j,\{g>t\} \subset \bigcup_{i} 5 B_{i}$ and

$$
\left|B_{i}\right| \leq \frac{C}{t^{\frac{1}{\theta_{2}-\theta_{1}}}}\left(\int_{B_{i}}|h| d y\right)^{\frac{\theta_{2}}{\theta_{2}-\theta_{1}}} .
$$

Here $c B$ denotes the ball with the same center as $B$ but $c$ times its radius. Thus

$$
|\{g>t\}| \leq \sum_{i} \frac{C}{t^{\frac{1}{\theta_{2}-\theta_{1}}}}\left(\int_{B_{i}}|h| d y\right)^{\frac{\theta_{2}}{\theta_{2}-\theta_{1}}} .
$$

Since $B_{i} \cap B_{j}=\varnothing$ for $i \neq j$ and $\frac{\theta_{2}}{\theta_{2}-\theta_{1}} \geq 1$, it follows that

$$
|\{g>t\}| \leq \frac{C}{t^{\frac{1}{\theta_{2}-\theta_{1}}}}\left(\int_{\mathbb{R}^{N}}|h| d y\right)^{\frac{\theta_{2}}{\theta_{2}-\theta_{1}}} .
$$


To introduce the truncation method, we need the following definition.

Definition 2 Let $l<m$, and $g$ be a real measurable function defined on $\mathbb{R}^{N}$. Consider $h_{l, m}: \mathbb{R} \rightarrow \mathbb{R}$ given by

$$
h_{l, m}(t)= \begin{cases}m-l & \text { if } m<t \\ t-l & \text { if } l<t \leq m, \\ 0 & \text { otherwise }\end{cases}
$$

and define the operator $T(l, m, \cdot)$ as follows

$$
T(l, m, g)(x)=h_{l, m}(g(x)) .
$$

The following lemma plays an important role in the proof of Theorem 3.

Lemma 8 Let $p, r \geq 1, \delta>0$, and $g \in L_{\text {loc }}^{1}\left(\mathbb{R}^{N}\right)$. Define $g_{k}=T\left(10^{k}, 10^{k+2},|g|\right)$ for $k \in \mathbb{Z}$. Assume that there exist a sequence of functions $\left(v_{k}\right) \subset L^{1}\left(\mathbb{R}^{N}\right)$ and a function $v \in L^{1}\left(\mathbb{R}^{N}\right)$ such that

$$
\left|\left\{g_{k}^{\sharp, \Delta}>t\right\}\right| \leq \frac{\left\|v_{k}\right\|_{L^{1}}^{r}}{t^{p}}, \quad \forall t>\delta, k \in \mathbb{Z}
$$

and

$$
\sum_{k \in \mathbb{Z}}\left|v_{k}\right| \leq v
$$

Then

$$
\int_{|g|>\lambda \delta}|g|^{p} d x \leq C\|v\|_{L^{1}}^{r},
$$

for positive constants $C$ and $\lambda$, depending only on $N$ and $p$.

Proof We first recall the following estimate [24, Estimate (22), p. 153]): Let $0<b<1$, $c>0$, and $f \in L_{\text {loc }}^{1}\left(\mathbb{R}^{N}\right)$. Then

$$
\left|\left\{M^{\Delta} f>\alpha, f^{\sharp, \Delta} \leq c \alpha\right\}\right| \leq \frac{2^{N} c}{1-b}\left|\left\{M^{\Delta} f>b \alpha\right\}\right|, \quad \forall \alpha>0 .
$$

Applying (4.5) with $f=g_{k}, b=\frac{1}{10}, \alpha=10^{k}$, and $0<c<\frac{1}{2}$, which depends only on $N$ and $p$, and is defined later, we have

$$
\left|\left\{M^{\Delta} g_{k}>10^{k}\right\}\right| \leq c 2^{N+1}\left|\left\{M^{\Delta} g_{k}>10^{k-1}\right\}\right|+\left|\left\{g_{k}^{\sharp, \Delta}>c 10^{k}\right\}\right|,
$$

which implies

$$
10^{k p}\left|\left\{M^{\Delta} g_{k}>10^{k}\right\}\right| \leq c 2^{N+1} 10^{k p}\left|\left\{M^{\Delta} g_{k}>10^{k-1}\right\}\right|+10^{k p}\left|\left\{g_{k}^{\sharp, \Delta}>c 10^{k}\right\}\right| .
$$

Take $k_{0} \in \mathbb{Z}$ such that $c 10^{k_{0}-2} \leq \delta<c 10^{k_{0}-1}$. Then (4.6) implies, for $m \geq k_{0}+1$,

$$
\begin{aligned}
\sum_{k_{0}}^{m} 10^{k p}\left|\left\{M^{\Delta} g_{k}>10^{k}\right\}\right| \leq & c 2^{N+1} \sum_{k_{0}}^{m} 10^{k p}\left|\left\{M^{\Delta} g_{k}>10^{k-1}\right\}\right| \\
& +\sum_{k_{0}}^{m} 10^{k p}\left|\left\{g_{k}^{\sharp, \Delta}>c 10^{k}\right\}\right| .
\end{aligned}
$$


We first establish a lower bound for the left hand side of (4.7). Since

$$
\left|\left\{M^{\Delta} g_{k}>10^{k}\right\}\right| \geq\left|\left\{g_{k}>10^{k}\right\}\right|,
$$

it follows from the definition of $M^{\Delta} g_{k}$ and $g_{k}$ that

$$
\sum_{k_{0}}^{m} 10^{k p}\left|\left\{M^{\Delta} g_{k}>10^{k}\right\}\right| \geq C_{N, p} \int_{10^{k_{0}+1}}^{10^{m+2}} t^{p-1}|\{|g|>t\}| d t \quad \forall m \geq k_{0}+1 .
$$

We next show an upper bound for the right hand side of (4.7). Using the theory of maximal functions (see e.g. [23, Theorem 1 in p. 5]), we have

$$
\sum_{k_{0}}^{m} 10^{k p}\left|\left\{M^{\Delta} g_{k}>10^{k-1}\right\}\right| \leq C_{N, p} \sum_{k_{0}}^{m} \int_{\mathbb{R}^{N}}\left|g_{k}\right|^{p} d x \leq C_{N, p} \int_{10^{k_{0}}}^{10^{m+2}} t^{p-1}|\{|g|>t\}| d t,
$$

for all $m \geq k_{0}+1$. The last inequality in (4.9) follows from the definition of $g_{k}$. On the other hand, since $r \geq 1$, it follows from (4.3) and the definition of $v_{k}$ that

$$
\sum_{k_{0}}^{m} 10^{k p}\left|\left\{g_{k}^{\sharp, \Delta}>c 10^{k}\right\}\right| \leq \frac{1}{c^{p}} \sum_{k_{0}}^{m}\left\|v_{k}\right\|_{L^{1}}^{r} \leq \frac{1}{c^{p}}\|v\|_{L^{1}}^{r} .
$$

Combining (4.9) and (4.10), we obtain

$$
\begin{aligned}
& c 2^{N+1} \sum_{k_{0}}^{m} 10^{k p}\left|\left\{M^{\Delta} g_{k}>10^{k-1}\right\}\right|+\sum_{k_{0}}^{m} 10^{k p}\left|\left\{g_{k}^{\sharp, \Delta}>c 10^{k}\right\}\right| \\
& \leq C_{N, p}\left(c 2^{N+1} \int_{10^{k_{0}}}^{10^{m+2}} t^{p-1}|\{|g|>t\}| d t+\frac{1}{c^{p}}\|v\|_{L^{1}}^{r}\right),
\end{aligned}
$$

which is an upper bound for the right hand side of (4.7).

From (4.7), (4.8), and (4.11), we have

$$
\int_{10^{k_{0}+1}}^{10^{m+2}} t^{p-1}|\{|g|>t\}| d t \leq C_{N, p}\left(c 2^{N+1} \int_{10^{k_{0}}}^{10^{m+2}} t^{p-1}|\{|g|>t\}| d t+\frac{1}{c^{p}}\|v\|_{L^{1}}^{r}\right) .
$$

Take $c$ such that $C_{N, p} c 2^{N+1}=1 / 2$. It follows from (4.12) that

$$
\int_{10^{k_{0}+1}}^{10^{m+2}} t^{p-1}|\{|g|>t\}| d t \leq C_{N, p}\left(\int_{10^{k_{0}}}^{10^{k_{0}+1}} t^{p-1}|\{|g|>t\}| d t+\frac{1}{c^{p}}\|v\|_{L^{1}}^{r}\right) .
$$

By (4.3) and (4.4), this implies

$$
\int_{10^{k_{0}+1}}^{10^{m+2}} t^{p-1}|\{|g|>t\}| d t \leq C_{N, p}\|v\|_{L^{1}}^{r} .
$$

Letting $m$ go to infinity, the conclusion follows. 
We are ready to give

Proof of Theorem 3 Let $k \in \mathbb{Z}$ be such that $10^{k} \geq \delta$. Define

$$
g_{k}=T\left(10^{k}, 10^{k+2},|g|\right)
$$

(see Definition 2). From (1.4), we have

$$
\begin{aligned}
& C_{N, p} \int_{B} \int_{B}\left|g_{k}(x)-g_{k}(y)\right|^{p} d x d y \leq|B|^{\frac{N+p}{N}} \\
& \times \int_{\substack{B \\
\delta<\left|g_{k}(x)-g_{k}(y)\right|<10 \delta}} \frac{\delta^{p}}{|x-y|^{N+p}} d x d y+\delta^{p}|B|^{2},
\end{aligned}
$$

for any ball $B$ of $\mathbb{R}^{N}$. Define

$$
h(x)=\int_{\substack{\mathbb{R}^{N} \\|g(y)-g(x)|>\delta}} \frac{\delta^{p}}{|y-x|^{N+p}} d y,
$$

and

$$
h_{k}(x)=h(x) \chi_{10^{k}<|g| \leq 10^{k+2}}(x) .
$$

Here $\chi_{A}$ denotes the characteristic function of a set $A \subset \mathbb{R}^{N}$. Since

$$
\int_{\substack{B \\ B}} \frac{\delta^{p}}{|x-y|^{N+p}} d x d y \leq 2 \int_{B} \chi_{10^{k}<|g| \leq 10^{k+2}} \int_{\substack{B \\|g(x)-g(y)|>\delta}} \frac{\delta^{p}}{|x-y|^{N+p}} d x d y,
$$

it follows from (4.13) that

$$
C_{N, p} g_{k}^{\sharp, \Delta}(x) \leq \sup _{Q}|Q|^{1 / N}\left(f_{Q}\left|h_{k}\right| d x\right)^{1 / p}+\delta,
$$

where the supremum is taken over all cubes $Q$ containing $x$. Applying Lemma 7, we obtain

$$
\left|\left\{g_{k}^{\sharp, \Delta}>t\right\}\right| \leq C_{N, p}\left\|h_{k}\right\|_{L^{1}}^{\frac{N}{N-p}} / t^{\frac{N p}{N-p}}, \quad \forall t>C_{N, p} \delta,
$$

According to Lemma 8, the conclusion follows.

\section{A general setting}

In this section we prove Propositions 4, 5, and 6 and present their applications. 


\subsection{Proof of Proposition 4}

We follows the approach used in the proof of Assertion (b) of [18, Theorem 1]. Since $F$ is a non-decreasing function, we have

$$
\int_{B} \int_{B} \frac{F(|g(x)-g(y)|)}{|x-y|^{N+p}} d x d y \geq \sum_{n \geq 0} \int_{\substack{B \\ 2^{-n}<|g(x)-g(y)| \leq 2^{-n+1}}} \frac{F\left(2^{-n}\right)}{|x-y|^{N+p}} d x d y .
$$

On the other hand,

$$
\begin{aligned}
\sum_{n \geq 0} \int_{\substack{B \\
2^{-n}<|g(x)-g(y)| \leq 2^{-n+1}}} \frac{F\left(2^{-n}\right)}{|x-y|^{N+p}} d x d y= & \sum_{n \geq 0} \int_{\substack{B \\
|g(x)-g(y)|>2^{-n}}} \frac{F\left(2^{-n}\right)}{|x-y|^{N+p}} d x d y \\
& -\sum_{n \geq 0} \int_{B} \int_{B} \frac{F\left(2^{-n-1}\right)}{|x-y|^{N+p}} d x d y \\
& -\int_{B} \int_{B} \underset{\substack{B(x)-g(y)\left|>2^{-n}\\
\right| g(x)-g(y) \mid>2}}{|x-y|^{N+p}} d x d y .
\end{aligned}
$$

This implies

$$
\begin{aligned}
& \sum_{n \geq 0} \int_{\substack{B \\
2^{-n}<|g(x)-g(y)| \leq 2^{-n+1}}} \frac{F\left(2^{-n}\right)}{|x-y|^{N+p}} d x d y \\
& =\sum_{n \geq 0} \int_{B} \int_{B} \frac{\left[F\left(2^{-n}\right)-F\left(2^{-n-1}\right)\right]}{|x-y|^{N+p}} d x d y \\
& -\int_{\substack{B(x)-g(y)\left|>2^{-n}\\
\right| g(x)-g(y) \mid>2}} \frac{F(1)}{|x-y|^{N+p}} d x d y .
\end{aligned}
$$

Combining (5.1) and (5.2) yields

$$
\begin{aligned}
& \int_{B} \int_{B} \frac{F(|g(x)-g(y)|)}{|x-y|^{N+p}} d x d y+\int_{B} \int_{B} \frac{F(1)}{|g(x)-g(y)|>2}|x-y|^{N+p} d x d y \\
& \geq \sum_{n \geq 0} \int_{\substack{B \\
|g(x)-g(y)|>2^{-n}}} \frac{\left[F\left(2^{-n}\right)-F\left(2^{-n-1}\right)\right]}{|x-y|^{N+p}} d x d y .
\end{aligned}
$$

Applying Theorem 1, we have

$$
|B|^{\frac{N+p}{N}} \quad \int_{B} \int_{B} \frac{1}{|x-y|^{N+p}} d x d y+|B|^{2} \gtrsim 2^{n p} \int_{B} \int_{B}|g(x)-g(y)|^{p} d x d y .
$$

$|g(x)-g(y)|>2^{-n}$ 
It follows that

$$
\begin{aligned}
& |B|^{\frac{N+p}{N}} \sum_{n \geq 0} \int_{\substack{B \\
|g(x)-g(y)|>2^{-n}}} \frac{\left[F\left(2^{-n}\right)-F\left(2^{-n-1}\right)\right]}{|x-y|^{N+p}} d x d y+\sum_{n \geq 0}\left[F\left(2^{-n}\right)-F\left(2^{-n-1}\right)\right]|B|^{2} \\
& \gtrsim \sum_{n \geq 0} 2^{n p}\left[F\left(2^{-n}\right)-F\left(2^{-n-1}\right)\right] \int_{B} \int_{B}|g(x)-g(y)|^{p} d x d y .
\end{aligned}
$$

On the other hand, we have

$$
\begin{aligned}
\sum_{n \geq 0} 2^{n p}\left[F\left(2^{-n}\right)-F\left(2^{-n-1}\right)\right] & =F(1)+\sum_{n \geq 1}\left(2^{n p}-2^{n p-p}\right) F\left(2^{-n}\right) \\
& \gtrsim F(1)+\int_{0}^{1} F(t) t^{-(p+1)} d t,
\end{aligned}
$$

since $F$ is non-decreasing, and

$$
\sum_{n \geq 0}\left[F\left(2^{-n}\right)-F\left(2^{-n-1}\right)\right]=F(1) .
$$

Combining (5.4), (5.5), and (5.6) yields

$$
\begin{aligned}
& |B|^{\frac{N+p}{p}} \sum_{n \geq 0} \int_{\substack{B \\
|g(x)-g(y)|>2^{-n}}} \frac{\left[F\left(2^{-n}\right)-F\left(2^{-n-1}\right)\right]}{|x-y|^{N+p}} d x d y+F(1)|B|^{2} \\
& \gtrsim\left(F(1)+\int_{0}^{1} F(t) t^{-(p+1)} d t\right) \int_{B} \int_{B}|g(x)-g(y)|^{p} d x d y .
\end{aligned}
$$

We deduce from (5.3) and (5.7) that

$$
\begin{aligned}
& \left(F(1)+\int_{0}^{1} F(t) t^{-(p+1)} d t\right) \int_{B} \int_{B}|g(x)-g(y)|^{p} d x d y \\
& \lesssim|B|^{\frac{N+p}{N}} \int_{B} \int_{B} \frac{F(|g(x)-g(y)|)}{|x-y|^{N+p}} d x d y+|B|^{\frac{N+p}{N}} \\
& \times \int_{B} \int_{B} \frac{F(1)}{|x-y|^{N+p}} d x d y+F(1)|B|^{2} . \\
& |g(x)-g(y)|>2
\end{aligned}
$$

Since $F$ is non-decreasing, this implies

$$
\begin{aligned}
& \left(F(1)+\int_{0}^{1} F(t) t^{-(p+1)} d t\right) \int_{B} \int_{B}|g(x)-g(y)|^{p} d x d y \\
& \lesssim|B|^{\frac{N+p}{N}} \int_{B} \int_{B} \frac{F(|g(x)-g(y)|)}{|x-y|^{N+p}} d x d y+F(1)|B|^{2} .
\end{aligned}
$$


5.2 Proofs of Propositions 5 and 6

Proof of Proposition 5 Applying the same method as in Lemma 6, but, using Proposition 4 instead of Theorem 1, we have

$$
\int_{Q}\left|g_{n}-g_{n, \varepsilon}\right|^{p} d x \lesssim \varepsilon^{p} \int_{\mathbb{R}^{N}} \int_{\mathbb{R}^{N}} \frac{F_{n}\left(\left|g_{n}(x)-g_{n}(y)\right|\right)}{|x-y|^{N+p}} d x d y+F_{n}(1)|Q| .
$$

for any cube $Q$ of $\mathbb{R}^{N}$. Here $g_{n, \varepsilon}$ is defined as in the proof of Lemma 6. It follows that

$$
\lim _{\varepsilon \rightarrow 0}\left(\limsup _{n \rightarrow \infty} \int_{Q}\left|g_{n}(x)-g_{n, \varepsilon}(x)\right|^{p} d x\right)=0 .
$$

Therefore, there exist a sub-sequence $\left(g_{n_{k}}\right)$ of $\left(g_{n}\right)$ and $g \in L^{p}\left(\mathbb{R}^{N}\right)$ such that $g_{n_{k}}$ converges to $g$ in $L_{\text {loc }}^{p}\left(\mathbb{R}^{N}\right)$ (see the proof of Theorem 2).

Proof of Proposition 6 The conclusion of Proposition 6 follows from Proposition 4 by applying the same method used in the proof of Theorem 3 (see (5.3)). The details of the proof are left for the reader.

\subsection{Applications}

In this section, we give some applications of Propositions 4, 5, and 6.

Set

$$
F_{\varepsilon}(t)= \begin{cases}\varepsilon t^{p+\varepsilon} & \text { if } 0 \leq t \leq 1, \\ \varepsilon & \text { otherwise. }\end{cases}
$$

Then $F_{\varepsilon}$ is non-decreasing, $\int_{0}^{1} F_{\varepsilon}(t) t^{-(p+1)} d t+F_{\varepsilon}(1)=1$, and $\lim _{\varepsilon \rightarrow 0} F_{\varepsilon}(t)=0$ for all $t>0$. As a consequences of Propositions 4, 5, and 6, we have

Corollary 1 Let $p \geq 1, B$ be a ball of $\mathbb{R}^{N}$, and $g \in L^{p}(B)$. Then there exists a constant $C>0$, depending only on $N$ and $p$, such that

$$
\begin{aligned}
& C \int_{B} \int_{B}|g(x)-g(y)|^{p} d x d y \\
& \leq|B|^{\frac{N+p}{N}} \int_{\substack{B \\
|g(x)-g(y)| \leq 1}} \frac{\varepsilon|g(x)-g(y)|^{p+\varepsilon}}{|x-y|^{N+p}} d x d y+|B|^{\frac{N+p}{N}} \int_{B} \int_{B} \frac{\varepsilon}{|g(x)-g(y)|>1} d x d y \\
& \quad+\varepsilon|B|^{2} .
\end{aligned}
$$

Corollary 2 Let $\left(g_{n}\right)$ be a sequence of functions in $L^{p}\left(\mathbb{R}^{N}\right)(1 \leq p<N)$ and $\left(\varepsilon_{n}\right)$ be a positive sequence converging to 0 . Assume that

$\liminf _{n \rightarrow \infty}\left(\int_{\substack{\mathbb{R}^{N} \\|g(x)-g(y)| \leq 1}} \frac{\varepsilon_{n}|g(x)-g(y)|^{p+\varepsilon_{n}}}{|x-y|^{N+p}} d x d y+\int_{\substack{\mathbb{R}^{N} \\|g(x)-g(y)|>1}} \frac{\varepsilon_{n}}{|x-y|^{N+p}} d x d y\right)<+\infty$. 
Then there exist a subsequence $\left(g_{n_{k}}\right)$ of $\left(g_{n}\right)$ and $g \in L^{p}\left(\mathbb{R}^{N}\right)$ such that $\left(g_{n_{k}}\right)$ converges to $g$ in $L_{\mathrm{loc}}^{p}\left(\mathbb{R}^{N}\right)$.

Corollary 3 Let $0<\varepsilon<1,1<p<N$ and $g \in L^{p}\left(\mathbb{R}^{N}\right)$. Assume that

$$
\int_{\substack{\mathbb{R}^{N} \\ g(x)-g(y) \mid \leq 1}} \frac{\varepsilon|g(x)-g(y)|^{p+\varepsilon}}{|x-y|^{N+p}} d x d y+\int_{\substack{\mathbb{R}^{N} \\|g(x)-g(y)|>1}} \int_{\mathbb{R}^{N}} \frac{\varepsilon}{|x-y|^{N+p}} d x d y<+\infty .
$$

Then $g \in L^{q}\left(\mathbb{R}^{N}\right)$ with $q=\frac{N p}{N-p}$ and there exist two positive constants $C$ and $\lambda$ depending only on $N$ and $p$, such that

$$
\begin{aligned}
& \left(\int_{|g|>\lambda \varepsilon}|g|^{q} d x\right)^{\frac{1}{q}} \\
& \leq C\left(\int_{\substack{\mathbb{R}^{N} \\
|g(x)-g(y)| \leq 1}} \frac{|g(x)-g(y)|^{p+\varepsilon}}{|x-y|^{N+p}} d x d y+\int_{\substack{\mathbb{R}^{N} \\
\mathbb{R}^{N} \\
|g(x)-g(y)|>1}} \frac{1}{|x-y|^{N+p}} d x d y\right)^{\frac{1}{p}} .
\end{aligned}
$$

\section{References}

1. Ambrosio, L.: Metric space valued functions of bounded variation. Ann. Scuola Norm. Sup. Pisa Cl. Sci. 17, 493-498 (1990)

2. Bourgain, J., Nguyen, H.-M.: A new characterization of Sobolev spaces. C. R. Acad. Sci. Paris 343, 7580 (2006)

3. Bourgain, J., Brezis, H., Mironescu, P.: Another look at Sobolev spaces. In: Menaldi, J.L., Rofman, E., Sulem, A. (eds.) Optimal Control and Partial Differential Equations. A Volume in Honour of A. Bensoussan's 60th Birthday, pp. 439-455. IOS Press, Amsterdam (2001)

4. Bourgain, J., Brezis, H., Mironescu, P.: Complements to the paper: Lifting, Degree, and Distributional Jacobian Revisited, personal communication (2004)

5. Brezis, H.: Analyse Fonctionnelle. Théorie et applications, Mathématiques appliquées pour la maîtrise. Dunod, Paris (2002)

6. Brezis, H., Nguyen, H.-M.: On the distributional Jacobian of maps from $\mathbb{S}^{N}$ into $\mathbb{S}^{N}$ in fractional Sobolev and Hölder spaces. Ann. Math. to appear (2010)

7. Brezis, H., Nguyen, H.-M.: On a new class of functions related to $V M O$, C. R. Acad. Sci. Paris, to appear (2010)

8. Evans, L.C., Gariepy, R.F.: Measure Theory and Fine Properties of Functions, Studies in Advanced Mathematics. CRC Press, Boca Raton, FL (1992)

9. Fefferman, C., Stein, E.: $H^{p}$ spaces of several variables. Acta. Math. 129, 137-193 (1972)

10. Franchi, B., Lu, G., Wheeden, R.: A relationship between Poincaré-type inequalities and representation formulas in spaces of homogeneous type. Int. Math. Res. Not. 1, 1-14 (1996)

11. Garofalo, N., Nhieu, D.-M.: Isoperimetric and Sobolev inequalities for Carnot-Carathéodory spaces and the existence of minimal surfaces. Commun. Pure Appl. Math. 49, 1081-1144 (1996)

12. Hajlasz, P., Koskela, P.: Sobolev met Poincaré. Memoirs Am. Math. Soc. 145, 1-101 (2000)

13. John, F., Nirenberg, L.: On functions of bounded mean oscillation. Commun. Pure Appl. Math. 14, 415426 (1961)

14. Korevaar, N., Schoen, R.: Sobolev spaces and harmonic maps for metric space targets. Commun. Anal. Geom. 1, 561-659 (1993)

15. Mazya, V.G.: Sobolev Spaces. Springer, Berlin (1985)

16. Nguyen, H.-M.: Some new characterizations of Sobolev spaces. J. Funct. Anal. 237, 689-720 (2006)

17. Nguyen, H.-M.: Г-convergence and Sobolev norms. C. R. Acad. Sci. Paris 345, 679-684 (2007) 
18. Nguyen, H.-M.: Further characterizations of Sobolev spaces. J. Eur. Math. Soc. 10, 191-229 (2008)

19. Nguyen, H.-M.: $\Gamma$-convergence, Sobolev norms, and BV functions, Duke Math. J., to appear (2010)

20. Ponce, A.C.: An estimate in the spirit of Poincaré's inequality. J. Eur. Math. Soc. 6, 1-15 (2004)

21. Reshetnyak, Y.: Sobolev classes of functions with values in a metric space. Sib. Math. J. 38, 567583 (1997)

22. Saloff-Coste, L.: A note on Poincaré, Sobolev, and Harnack inequalities. Int. Math. Res. Not. 2, 27-38 (1992)

23. Stein, E.: Singular Integrals and Differentiability Properties of Functions, Princeton Mathematical Series, vol. 30. Princeton University Press, Princeton (1970)

24. Stein, E.: Harmonic Analysis: Real-Variable Methods, Orthogonality, and Oscillatory Integrals, vol. 43. Princeton University Press, Princeton (1993) 